\section{Solar wind dynamic pressure and electric field as the main factors controlling Saturn's aurorae} J. T. Steinberg ${ }^{5}$, B. L. Barraclough ${ }^{5}$, A. J. Coates ${ }^{6}$, J.-C. Gérard ${ }^{7}$, D. Grodent ${ }^{7}$, W. S. Kurth ${ }^{8}$, D. G. Mitchell ${ }^{9}$, A. M. Rymer ${ }^{6}$ \& D. T. Young

${ }^{1}$ Southwest Research Institute, Culebra Road, San Antonio, Texas 78288, USA ${ }^{2}$ Boston University, 725 Commonwealth Avenue, Boston, Massachusetts 02215, USA

${ }^{3}$ Blackett Laboratory, Imperial College of Science and Technology, London SW7 2BZ, UK

${ }^{4}$ The University of Michigan, Space Research Building, Ann Arbor, Michigan 48109, USA

${ }^{5}$ Los Alamos National Laboratory, Los Alamos, New Mexico 87545, USA

${ }^{6}$ University College London, Mullard Space Science Laboratory, Surrey RH5 6NT, UK

${ }^{7}$ Université de Liège, 17 avenue du 6 aout, B4000 Liège, Belgium

${ }^{8}$ Department of Physics and Astronomy, The University of Iowa, Iowa City, Iowa 52242, USA

${ }^{9}$ Applied Physics Laboratory, Johns Hopkins University, 11100 Johns Hopkins Road, Laurel, Maryland 20723, USA

The interaction of the solar wind with Earth's magnetosphere gives rise to the bright polar aurorae and to geomagnetic storms ${ }^{1}$, but the relation between the solar wind and the dynamics of the outer planets' magnetospheres is poorly understood. Jupiter's magnetospheric dynamics and aurorae are dominated by processes internal to the jovian system ${ }^{2}$, whereas Saturn's magnetosphere has generally been considered to have both internal and solar-wind-driven processes. This hypothesis, however, is tentative because of limited simultaneous solar wind and magnetospheric measurements. Here we report solar wind measurements, immediately upstream of Saturn, over a one-month period. When combined with simultaneous ultraviolet imaging ${ }^{3}$ we find that, unlike Jupiter, Saturn's aurorae respond strongly to solar wind conditions. But in contrast to Earth, the main controlling factor appears to be solar wind dynamic pressure and electric field, with the orientation of the interplanetary magnetic field playing a much more limited role. Saturn's magnetosphere is, therefore, strongly driven by the solar wind, but the solar wind conditions that drive it differ from those that drive the Earth's magnetosphere.

In the case of Earth's auroral activity and associated magnetic substorms, the north-south component of the interplanetary magnetic field is especially important. A southward interplanetary magnetic field allows efficient dayside reconnection between the Earth's and the interplanetary magnetic field, and is an important driver of substorms, whereas a northward field results in highlatitude reconnection and significantly weaker control of magnetospheric dynamics by the solar wind ${ }^{1}$. Solar wind shocks drive auroral activity, regardless of the interplanetary magnetic field orientation, and the magnitude of this effect correlates well with the solar wind speed and magnetic field strength. However, the influence of shocks is much more pronounced when the interplanetary magnetic field is southward ${ }^{4}$.

In contrast, Jupiter's rapid rotation and strong magnetic field, together with a large, internal plasma source from its satellite, Io, drive radial, outward transport of plasma and magnetospheric currents. These processes are the primary source of dynamics (ref. 2, and references therein) and the aurorae 5 . Despite this, the solar wind does exert some influence on Jupiter's magnetosphere ${ }^{6-9}$. On the basis of the size of the planet's magnetosphere and the observed ease with which its magnetopause is pushed inward and outward by changing solar wind conditions, it has been suggested that dynamic pressure is the main solar wind driver, rather than the orientation of the interplanetary magnetic field ${ }^{10,11}$. Saturn, like Jupiter, is a rapidly rotating planet and has significant internal plasma sources. However, its magnetic field and internal plasma sources are weaker than Jupiter's. Observations ${ }^{12}$ by the Voyager spacecraft showed a significant degree of solar wind control, although the origin of these correlations was unclear. In the case of the Voyager studies, kilometric radio emissions were used as a proxy for magnetospheric dynamics.

Between 10 and 30 January 2004, the Cassini spacecraft measured the solar wind upstream of Saturn's magnetosphere while the Hubble Space Telescope (HST) made observations of Saturn's ultraviolet aurorae roughly once every other day ${ }^{3}$. The Cassini data discussed here come primarily from the CAPS instrument (Cassini Plasma Spectrometer Subsystem, providing ion and electron data from $1 \mathrm{eV}$ to $50 \mathrm{keV}$; ref. 13) and from the magnetometer. During this period, Cassini solar wind ion measurements were largely continuous ( $81 \%$ duty cycle) and interrupted only by occasional downlinks of data to Earth. Cassini also made measurements of energetic particle flux, local plasma waves and auroral radio emissions from Saturn ${ }^{14}$.

During this period, the Cassini spacecraft was at a range of $(78 \pm 5) \times 10^{6} \mathrm{~km}$ from Saturn, over the post-dawn side of the planet, and $(31 \pm 2) \times 10^{6} \mathrm{~km}$ upstream. Although this distance seems large for an upstream solar wind monitor, the Saturn-Sunspacecraft angle was under $3.3^{\circ}$. Studies of Cassini and Galileo data, obtained near Jupiter, indicate that a spacecraft may act as a viable upstream monitor when the planet-Sun-spacecraft angle is less than $\sim 20^{\circ}$ (ref. 15). Moving at nominally $500 \mathrm{~km} \mathrm{~s}^{-1}$, it would take a spherically symmetric disturbance in the solar wind $17 \mathrm{~h}$ to travel from Cassini to Saturn.

Figure 1 shows the solar wind magnetic field, ion speed and

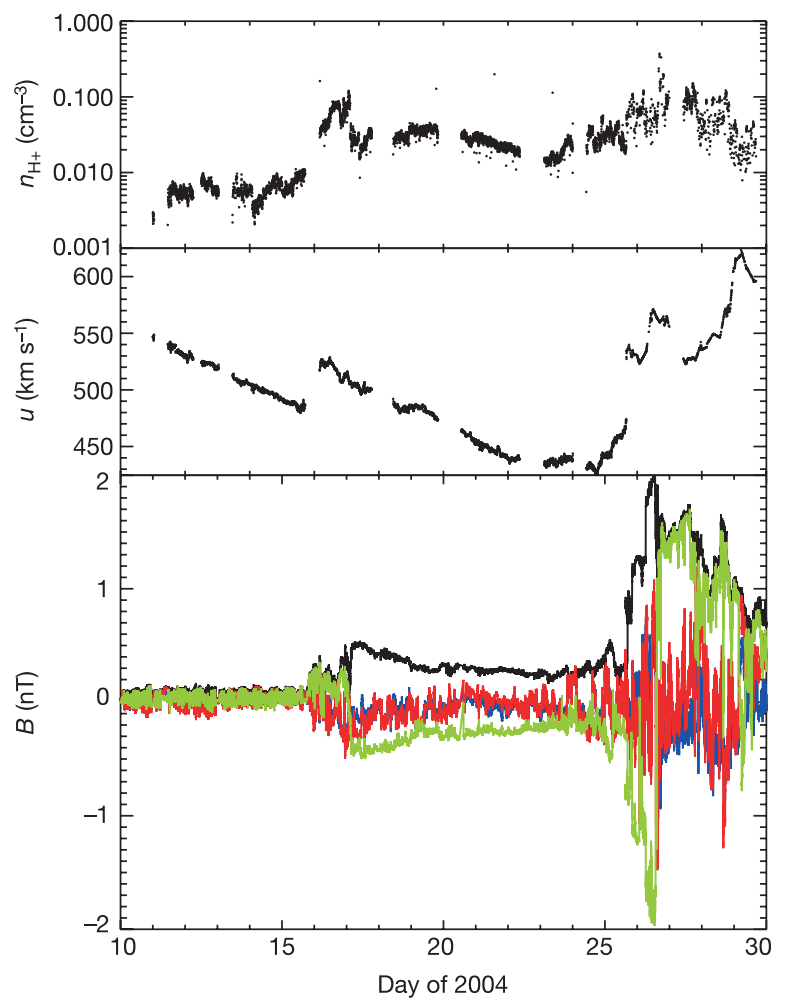

Figure 1 Solar wind conditions measured by Cassini. The top panel shows proton density, the middle panel the flow speed, and the lower panel the magnetic field. The black line shows the magnitude of the magnetic field. $B_{x}$ is shown in blue, $B_{y}$ in green and $B_{z}$ in red. The magnetic field is in planetary coordinates, a right-handed system with $z$ parallel to the axis of rotation (and magnetic moment), and the Sun in the $+x / z$ half-plane. 
proton density. The solar wind speed is characterized by periods of gradual decrease followed by shocks on 15 and 25 January. Enhanced ion densities are associated with these shocks, as are enhancements in energetic particle flux and low frequency plasma waves. Energetic particle, wave and magnetic field data indicate another shock on 1 January, when the spacecraft orientation did not allow direct measurements of core solar wind protons. Following the 25 January shock, the plasma density remained high and variable until the end of the month, the magnetic field strength was enhanced and several subsequent shocks were encountered. These solar wind disturbances are consistent with corotating interaction regions. A bimodal solar wind structure as observed here is expected during the declining phase of the solar cycle magnetic sector boundaries, with a reversal of the dominant $B_{y}$ component that occurred on 17 and 26 January. ( $B_{y}$ indicates the $y$ component of the magnetic field; see Fig. 1 legend for coordinate system.) During the month of observations, the north-south component of the interplanetary magnetic field was weak, and the field was primarily in the east-west direction.

We have used Cassini measurements to estimate conditions at Saturn with a one-dimensional, numerical magneto-hydrodynamic model ${ }^{16}$. This model assumes spherical symmetry and propagates the measurements outward. It accounts for the fact that shocks propagate faster than the ambient flow speed, as well as for any evolution of the solar wind during the final $0.25 \mathrm{AU}$ of its trip to Saturn. As the model assumes spherical symmetry, the threedimensional structure of the solar wind may cause errors in the modelled propagation time. We expect that our estimated arrival times may be systematically late by up to roughly $10 \mathrm{~h}$, but cannot be more exact owing to the unconstrained, three-dimensional structure of the solar wind.

According to this model, the shocks observed on 15 and 25 January reached Saturn at 12:45 UTC, 16 January, and 08:00 UTC,

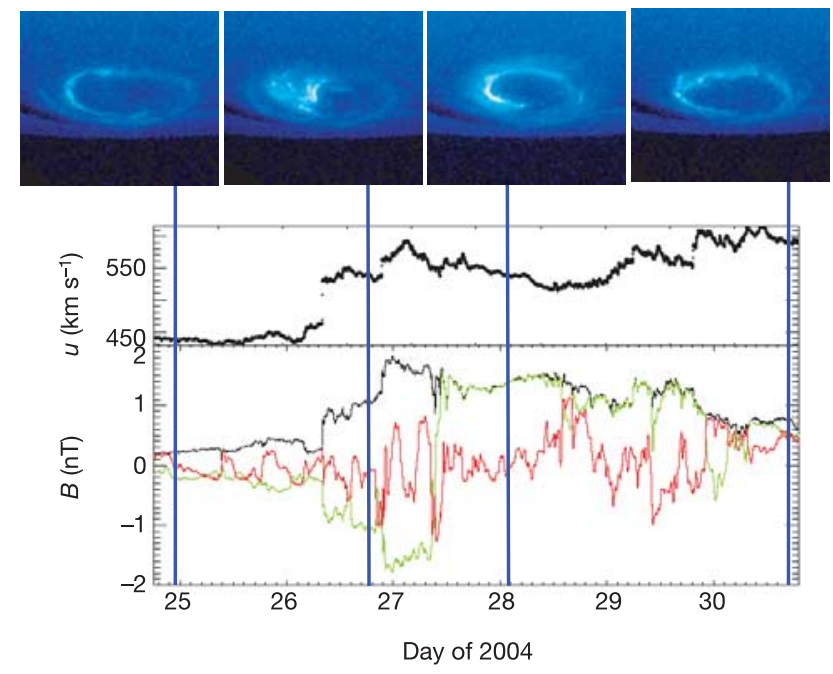

Figure 2 Comparison between HST images and solar wind conditions propagated to Saturn for the period 25-30 January 2004. For details of the HST images, see ref. 3. Thick blue vertical lines indicate the times of the four HST images shown above. The solar wind conditions are presented in the same format as Fig. 1, but density and the $B_{x}$ component are omitted for clarity. In one-dimensional, time-dependent magnetohydrodynamics with a uniform upstream boundary condition, the $x$ component cannot be accurately modelled. The modelled propagation times may be systematically long by as much as $10 \mathrm{~h}$. Non-radial orientation of the solar wind structures, typical of corotating interaction regions, could cause them to reach Saturn roughly five hours earlier than spherically symmetric calculations would suggest. Solar rotation and the $3.3^{\circ}$ difference between the spacecraft's and Saturn's heliocentric longitude could also move arrival times earlier by approximately $6 \mathrm{~h}$. Overall, the estimated arrival times may be systematically late by up to roughly $10 \mathrm{~h}$.
26 January. Figure 2 shows the propagated plasma speed and the magnetic field, along with four of the HST images. The first image, $32 \mathrm{~h}$ before the arrival of the 25 January shock, is typical of other images taken during undisturbed solar wind conditions. The second image, $11 \mathrm{~h}$ after the shock arrival $(+11 \mathrm{~h})$, shows a brightened and partially filled auroral oval. In the third image, at $+42 \mathrm{~h}$, the oval has shifted to higher latitudes while remaining atypically bright. In the final image, at $+107 \mathrm{~h}$, the aurora has returned to a more typical state. The response to the 15 January shock appears similar, although both the shock and the auroral response were weaker, and the first image obtained after the shock was at $+40 \mathrm{~h}$ (ref. 3 ).

Figure 3 shows a plot of propagated solar wind dynamic pressure against the input auroral power, estimated from the total ultraviolet brightness ${ }^{3}$. There is a very good correlation between auroral power and both the solar wind dynamic pressure, $\rho u^{2}(r=0.93)$, and the convection electric field, $\mathbf{u} \times \mathbf{B}(r=0.97)$. (Here $\rho$ is the mass density, $u$ the flow velocity, $r$ the linear correlation coefficient, $\mathbf{u}$ the flow velocity vector, and $\mathbf{B}$ the magnetic field vector.) These high correlation coefficients are, in part, a statistical artefact of the 25 January event, which produced the two unusually high-power points. However, this event is not the sole source of the correlations. Using only those observations with an auroral power below $5 \times 10^{10} \mathrm{~W}$ (that is, using only the lower-power half of the measurements and thus excluding the unusual events), we obtain correlation coefficients of 0.62 and 0.92 , respectively.

Correlations with auroral power also show a lack of control by the direction of the interplanetary magnetic field. In the case of Earth's magnetosphere, the dynamics correlate well with a gated solar wind electric field, $\mathbf{u} \times \mathbf{B} \cos ^{4}(\theta / 2)$, where $\theta$ is the projected angle between the interplanetary magnetic field and the planet's magnetic moment ${ }^{17}$. The $\theta$ term accounts for efficient, low-latitude reconnection when the interplanetary magnetic field is parallel to the planet's magnetic moment. For the January measurements, the correlation coefficient with this parameter is 0.74 . This is a significantly lower value than that obtained without the $\theta$ term, suggesting that the orientation of the interplanetary magnetic field is not a controlling factor.

At the same time, the interplanetary magnetic field during January was primarily in the $y$ direction, while the north-south component was weak and varying, with $\theta=91 \pm 32^{\circ}$. An alternative explanation is that the $\cos ^{4}$ gating function is not well-suited for the interaction between the solar wind and Saturn's magnetosphere. This relation was derived for the Earth and for a wide variety of solar wind magnetic field orientations, including the predominantly southward conditions that produce a strong magnetospheric

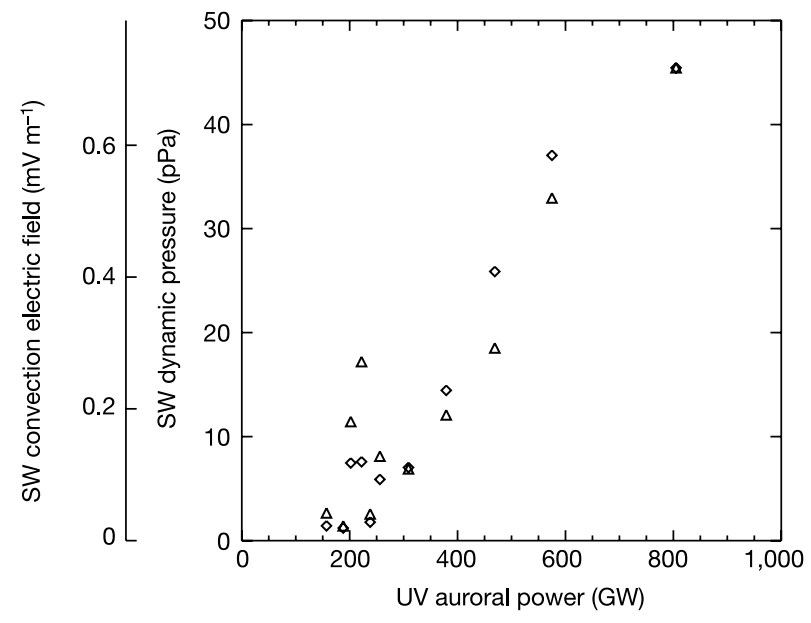

Figure 3 Correlation between HST-derived auroral input power and solar wind conditions. Triangles show the solar wind (SW) dynamic pressure, $\rho u^{2}$; diamonds show the solar wind convection electric field, $|\mathbf{u} \times \mathbf{B}|$. 
response. It is possible that the most appropriate gating function for values of $\theta$ near $90^{\circ}$ differs from the $\cos ^{4}$ relation best suited for the full range of $\theta$.

It is interesting to note that the January observations occurred near southern summer, and that the average interplanetary magnetic field is almost purely azimuthal at Saturn's distance from the Sun. As a result, the dominant component of the field was perpendicular to Saturn's magnetic moment. Solar wind control of Saturn's magnetosphere could be significantly different near the equinoxes, when Saturn's axial tilt results in a $64.5^{\circ}$ angle between the dominant, azimuthal field component and Saturn's magnetic moment. This would be a manifestation of a well-documented phenomenon at Earth $^{18}$, but made more extreme owing to the alignment between Saturn's magnetic moment and spin axis.

Overall, the January Cassini and HST measurements indicate that Saturn's magnetosphere, although similar in some respects to those of the Earth and Jupiter, is not simply an intermediate case. It resembles Earth's magnetosphere in that its auroral dynamics are strongly driven by solar wind conditions. But it differs from Earth's interaction with the solar wind by virtue of an evidently weak influence of the direction of the interplanetary magnetic field. This may either be a characteristic of Saturn's magnetosphere, or be a result of differences between the solar wind at $10 \mathrm{AU}$ and $1 \mathrm{AU}$ (specifically, the weaker north-south component of the solar wind magnetic field). The latter possibility suggests that Saturn's magnetospheric response to the solar wind may be primarily driven by solar wind shocks, a process that is observed at the Earth ${ }^{2}$ but generally overshadowed by the strong response of Earth's magnetosphere to the orientation of the interplanetary magnetic field. This apparent insensitivity to the magnetic field orientation and sensitivity to solar wind dynamic pressure and/or motional electric field make Saturn's magnetosphere resemble that of Jupiter more than that of Earth. In contrast to Jupiter, where the influence of the solar wind is weak and the aurorae are largely due to internal processes, the solar wind plays a controlling role in Saturn's auroral dynamics.

Received 9 September; accepted 27 December 2004; doi:10.1038/nature03333.

1. Dungey, J. W. Interplanetary magnetic field and the auroral zone. Phys. Rev. Lett. 6, 47-48 (1961).

2. Hill, T. W., Dessler, A. J. \& Goertz, C. K. in Physics of the Jovian Magnetosphere (ed. Dessler, A. J.) 353-394 (Cambridge Univ. Press, Cambridge, 1983).

3. Clarke, J. T. et al. Morphological differences of Saturn's ultraviolet aurorae and those of Earth and Jupiter. Nature doi:10.1038/nature03331 (this issue).

4. Meurant, M., Gerard, J.-C., Blockx, C., Hubert, B. \& Coumans, V. Propagation of electron and proton shock-induced aurora and the role of the interplanetary magnetic field and solar wind. J. Geophys. Res 109, A10210, doi:10.1029/2004JA010453 (2004).

5. Cowley, S. W. H. \& Bunce, E. J. Origin of the main auroral oval in Jupiter's coupled magnetosphereionosphere system. Planet. Space Sci. 49, 1067-1088 (2001).

6. Zarka, P. \& Genova, F. Low frequency jovian emissions and solar wind magnetic sector structure. Nature 306, 767-768 (1983)

7. Desch, M. D. \& Barrow, C. H. Direct evidence for solar wind control of Jupiter's hectometric wavelength radio emission. J. Geophys. Res. 89, 6819-6823 (1984).

8. Gurnett, D. A. et al. Control of Jupiter's radio emissions and aurorae by the solar wind. Nature 415 , 985-987 (2002).

9. Kivelson, M. G. \& Southwood, D. J. First evidence of IMF control of Jovian magnetospheric boundary locations: Cassini and Galileo magnetic field measurements compared. Planet. Space Sci. 51, 891-898 (2003).

10. Southwood, D. J. \& Kivelson, M. G. A new perspective concerning the influence of the solar wind on the Jovian magnetosphere. J. Geophys. Res. 106, 6123-6130 (2001).

11. Cowley, S. W. H. \& Bunce, E. J. Modulation of Jupiter's main auroral oval emissions by solar wind induced expansions and compressions of the magnetosphere. Planet. Space Sci. 51, 57-79 (2003).

12. Desch, M. D. Evidence for solar wind control of Saturn radio emission. J. Geophys. Res. 87, 4549-4554 (1982).

13. Young, D. T. et al. Cassini plasma spectrometer investigation. Space Sci. Rev. (in the press).

14. Kurth, W. S. et al. An Earth-like correspondence between Saturn's auroral features and radio emission. Nature doi:10.1038/nature03334 (this issue)

15. Hanlon, P. G. et al. On the evolution of the solar wind between 1 and $5 \mathrm{AU}$ at the time of the CassiniJupiter flyby: multi spacecraft observations of ICMEs including the formation of a merged interaction region. J. Geophys. Res. 109, A09S03, doi:10.1029/2003JA010112 (2004).

16. Tóth, G. General code for modeling MHD flows on parallel computers: Versatile advection code. Astrophys. Lett. Commun. 34, 245-250 (1996).

17. Akasofu, S.-I. The solar wind-magnetosphere energy coupling and magnetospheric disturbances. Planet. Space Sci. 28, 495-509 (1980).

18. Russell, C. T. \& McPherron, R. L. Semiannual variation of geomagnetic activity. J. Geophys. Res. 78, 92-108 (1973).
Acknowledgements F.J.C., B.L.B., J.T.S. and D.T.Y. were supported by NASA through a Jet Propulsion Laboratory contract with SWRI; D.G.M., K.C.H. and W.S.K. were supported through other NASA/JPL contracts; P.G.H. was supported by a PPARC-UK quota studentship; and J.C.G. and D.G. were supported by the Belgian Foundation for Scientific Research (FNRS) and the PRODEX program of the ESA. This work is based on observations with the NASA/ESA Hubble Space Telescope, obtained at the Space Telescope Science Institute, which is operated by the AURA, Inc., for NASA.

Competing interests statement The authors declare that they have no competing financial interests.

Correspondence and requests for materials should be addressed to F.J.C. (fcrary@swri.edu).

\section{An Earth-like correspondence between Saturn's auroral features and radio emission}

\section{W. S. Kurth ${ }^{1}$, D. A. Gurnett ${ }^{1}$, J. T. Clarke ${ }^{2}$, P. Zarka ${ }^{3}$, M. D. Desch ${ }^{4}$, M. L. Kaiser ${ }^{4}$, B. Cecconi ${ }^{1}$, A. Lecacheux ${ }^{3}$, W. M. Farrell ${ }^{4}$, P. Galopeau ${ }^{5}$, J.-C. Gérard ${ }^{6}$, D. Grodent ${ }^{6}$, R. Prangé ${ }^{3}$, M. K. Dougherty ${ }^{7}$ \& F. J. Crary ${ }^{8}$}

${ }^{1}$ Department of Physics and Astronomy, The University of Iowa, Iowa City, Iowa 52242, USA

${ }^{2}$ Boston University, 725 Commonwealth Avenue, Boston, Massachusetts 02215, USA

${ }^{3}$ Space Research Department, Observatoire de Paris, 92195 Meudon, France ${ }^{4}$ NASA/Goddard Space Flight Center, Greenbelt, Maryland 20771, USA ${ }^{5}$ CETP/UVSQ, 78140 Velizy, France

${ }^{6}$ LPAP, Université de Liège, allée du 6 aout, 17, B-4000 - Liège, Belgium ${ }^{7}$ Blackett Laboratory, Imperial College of Science and Technology, London SW7 2BZ, UK

${ }^{8}$ Southwest Research Institute, Culebra Road, San Antonio, Texas 78288, USA

Saturn is a source of intense kilometre-wavelength radio emissions that are believed to be associated with its polar aurorae ${ }^{1,2}$, and which provide an important remote diagnostic of its magnetospheric activity. Previous observations implied that the radio emission originated in the polar regions, and indicated a strong correlation with solar wind dynamic pressure ${ }^{1,3-7}$. The radio source also appeared to be fixed near local noon and at the latitude of the ultraviolet aurora ${ }^{1,2}$. There have, however, been no observations relating the radio emissions to detailed auroral structures. Here we report measurements of the radio emissions, which, along with high-resolution images of Saturn's ultraviolet auroral emissions ${ }^{8}$, suggest that although there are differences in the global morphology of the aurorae, Saturn's radio emissions exhibit an Earth-like correspondence between bright auroral features and the radio emissions. This demonstrates the universality of the mechanism that results in emissions near the electron cyclotron frequency narrowly beamed at large angles to the magnetic field ${ }^{9,10}$.

The first studies of Saturn's primary radio emission (Saturn kilometric radiation, SKR) were by the Voyager spacecraft in the early $1980 \mathrm{~s}^{1,3}$. This emission had some features that were more like those of Earth than of Jupiter: the SKR source appears to be fixed in local time, favouring a region near local late morning or noon, but varying with source latitude ${ }^{2}$. It is also strongly correlated with solar wind parameters, such as the dynamic pressure ${ }^{7}$. However, the SKR also has some features that are more like those of Jupiter than of Earth: it was found to be strongly modulated by planetary rotation, despite the fact that models of Saturn's magnetic field are axisymmetric $^{11}$. This has suggested an as-yet-unobserved 'active sector' or possible 'magnetic anomaly' rotating with the planet ${ }^{12,13}$.

The measurements shown here are primarily from the radio 